\title{
Cardiomiopatia Chagásica Na Amazônia Brasileira: Baixa Prevalência Ou Subdiagnóstico?
}

\section{Chagas Cardiomyopathy in the Brazilian Amazon Region: Low Prevalence or Underdiagnosis?}

Jessica Vanina Ortiz, ${ }^{1}$ Katia do Nascimento Couceiro, ${ }^{1}$ Susan Smith Doria, ${ }^{1 \oplus}$ Débora Raysa Teixeira de Sousa, ${ }^{1}$ Henrique Manuel Condinho da Silveira, ${ }^{2}$ Norival Kesper Junior, ${ }^{3}$ Maria das Graças Vale Barbosa Guerra, ${ }^{1,4}$ Jorge Augusto de Oliveira Guerra, ${ }^{1,4}$ João Marcos Bemfica Barbosa-Ferreira ${ }^{1,5} \bullet$

Programa de Pós-Graduação em Medicina Tropical, Escola de Ciências da Saúde, Universidade do Estado do Amazonas, ${ }^{1}$ Manaus, AM - Brasil Instituto de Higiene e Medicina Tropical, ${ }^{2}$ Lisboa - Portugal

Hospital das Clínicas da FMUSP-LIM49, ${ }^{3}$ São Paulo, SP - Brasil

Fundação de Medicina Tropical Dr. Heitor Vieira Dourado, ${ }^{4}$ Manaus, AM - Brasil

Fundação Hospital do Coração Francisca Mendes, ${ }^{5}$ Manaus, AM - Brasil

\section{Introdução}

A doença de Chagas (DC) foi descoberta em 1909 pelo médico brasileiro Carlos Chagas, que descreveu o agente etiológico - um protozoário flagelado denominado Trypanossoma cruzi - suas características morfológicas e seu ciclo de vida e de transmissão, bem como manifestações clínicas da doença. ${ }^{1}$ Apesar de os insetos triatomíneos serem os vetores primários de transmissão da DC, a via oral por meio de alimentos contaminados foi considerada o meio principal de transmissão na Amazônia brasileira.

A doença apresenta duas fases clínicas: uma infecção aguda, predominantemente assintomática com elevada parasitemia, e uma infecção crônica dividida em forma indeterminada assintomática ou forma sintomática (digestiva ou cardíaca). ${ }^{2}$

A cardiomiopatia chagásica crônica (CCC) é caracterizada por uma cardiomiopatia dilatada causada por uma inflamação crônica, e é a principal causa de morte entre pacientes com cardiomiopatia não isquêmica na América Latina..$^{3,4}$ A Organização Mundial da Saúde estima que haja oito milhões de pessoas infectadas em todo o mundo, e aproximadamente 232 mil pessoas com CCC no Brasil. ${ }^{2}$ Há poucos estudos publicados com indivíduos com DC crônica e CCC na região da Amazônia brasileira. O primeiro relato de casos crônicos foi realizado em 1977, ${ }^{5}$ e o do primeiro caso de CCC em 2003. ${ }^{6}$ Xavier et al. ${ }^{7}$ e Ferreira et al. ${ }^{8}$ também relataram novos casos de DC crônica e CCC. Em dez anos, nenhum estudo atualizou as características epidemiológicas de CCC na região.

\section{Palavras-chave}

Doença de Chagas; Insuficiência Cardíaca; Cardiomiopatia Chagásica.

Correspondência: João Marcos B. B. Ferreira •

Universidade do Estado do Amazonas - Av. Carvalho Leal, 1777. CEP 69065001, Manaus, AM - Brasil

Email: jmbemfica@hotmail.com

Artigo recebido em 25/11/2020, revisado em 08/04/2021, aceito em $12 / 05 / 2021$

DOI: https://doi.org/10.36660/abc.20201236
O diagnóstico de DC crônica é feito por dois testes sorológicos diferentes: ensaios imunoenzimáticos (ELISA), imunofluorescência indireta (IFI). Exame parasitológico, teste de biologia molecular, e western blot são usados como métodos complementares. Técnicas de biologia molecular têm sido amplamente utilizadas para identificar a linhagem do $T$. cruzi circulante na região., ${ }^{2,9} \mathrm{O}$ presente estudo teve como objetivo avaliar a prevalência de DC em pacientes com cardiomiopatia dilatada de causa desconhecida na região da Amazônia brasileira para tentar responder as seguintes perguntas: a CCC está sendo subdiagnosticada? Ou, existe uma baixa prevalência da doença em nossa região?

\section{Métodos}

\section{Locais do estudo}

Este estudo foi um estudo transversal conduzido no Hospital Universitário Francisca Mendes (Unidade de Cardiologia) e na Fundação de Medicina Tropical Doutor Heitor Vieira Dourado (doenças infecciosas / unidade de doença de Chagas), dois centros de atenção terciária especializados no estado do Amazonas, Brasil.

\section{População do estudo}

Os participantes foram recrutados de julho 2017 a julho 2018 e foram considerados elegíveis se apresentassem: 1) idade $\geq 18$ anos; 2) perfil cardíaco de cardiomiopatia dilatada idiopática, em que qualquer outra causa de cardiomiopatia já tiver sido excluída; 3) resultado anormal no ecocardiograma (fração de ejeção do ventrículo esquerdo [FEVE] reduzido e/ou alterações no segmento) e/ou no eletrocardiograma (bloqueio de ramo, bloqueio atrioventricular, ou arritmias).

Os seguintes fatores de risco epidemiológicos foram considerados: 1) ser originário da região da Amazônia brasileira; 2) ser originário de áreas rurais; 3) ter hábito de ir à floresta; e 4) consumir frutos de palmeiras e/ou carne de animais silvestres.

Pacientes com qualquer evidência de cardiomiopatia isquêmica ou congênita, ou de doença valvular cardíaca não foram elegíveis para inclusão. Ainda, os participantes que relataram ter viajado a outra região brasileira ou a um país estrangeiro foram excluídos do estudo. 


\section{Coleta de dados e de amostras de sangue}

Todos os pacientes que preencheram os critérios de inclusão foram convidados a participar e, aqueles que aceitaram, assinaram o termo de consentimento. Os participantes foram primeiramente avaliados usando um questionário clínico e epidemiológico. Em seguida, as amostras de sangue foram coletadas, centrifugadas, separadas e armazenadas a $-20^{\circ} \mathrm{C}$.

\section{Exames sorológicos}

O diagnóstico de DC foi realizado por ELISA (Chagatest ELISA recombinante v. 4.0, Wiener Laboratorios, Argentina) e imunofluorescência indireta (Imuno-CON Chagas, WAMA Diagnostica, Brasil), seguindo-se as instruções dos fabricantes.

Para resultados indeterminados, utilizou-se o teste de immunoblotting para análise de antígenos secretados e excretados por tripomastigota (TESA, do inglês trypomastigote excreted-secreted antigen), o TESA-blot. As frações de TESA foram obtidas a partir do sobrenadante de células MK2 infectadas pela cepa Y de T. cruzi, seguindo o protocolo descrito previamente. ${ }^{10}$

\section{Detecção e caracterização molecular do T. cruzi}

A extração de DNA foi realizada a partir de sangue periférico, seguindo-se o protocolo do kit comercial PureLink ${ }^{\circledR}$ Genomic DNA Mini (Invitrogen, Life Technologies, California, EUA). Este método foi realizado como um teste complementar para soros com resultados indeterminados nos exames sorológicos, para aumentar a acurácia diagnóstica. ${ }^{11}$

As amostras foram submetidas à tipagem do DNA mitocondrial pela análise de polimorfismos nos genes que codificam a subunidade II(COII). ${ }^{12}$ Os produtos de amplificação de PCR foram purificados utilizando-se o sistema Wizard SV Gel and PCR Clean-up System kit (Promega) e o sequenciamento realizado pelo sequenciador de DNA ABI 3130 (Applied Biosystems). Seguimos o protocolo do kit de sequenciamento BigDye Terminator v3.1 (Applied Biosystems) e sequências das cepas padrões: Tcl (Silvio X10 cl1), Tcll (Esmeraldo cl3), Tclll (M6241 cl6), TcIV (CANIII cl1), TcV (Mn cl2), e TcVI (CL Brener). A análise de evolução foi conduzida no MEGA X. ${ }^{13}$

\section{Aspectos éticos}

O estudo foi aprovado pelo comitê de ética em pesquisa da Fundação de Medicina Tropical Doutor Heitor Vieira Dourado (Manaus, AM, Brasil) (número 69904017.9.0000.00052.191.571/28, julho 2017), em concordância com a resolução 466/12 do Conselho Nacional de Saúde e diretrizes éticas da Declaração de Helsinki de 1975.

\section{Apresentação e análise dos dados}

Os dados clínicos e epidemiológicos foram organizados utilizando o Microsoft Excel 2016 e a análise descritiva realizada utilizando o Stata/MP 13.0. As variáveis categóricas foram descritas em frequências e proporções (\%), e as variáveis contínuas em médias e desvios padrões (DP).

\section{Resultados}

\section{Prevalência da doença de Chagas}

Foram incluídos 53 pacientes, e oito foram excluídos - um morreu antes da coleta de sangue, dois comentaram ter morado em outro local, e cinco não compareceram à coleta de sangue.

Nas 45 amostras viáveis, foram realizados os dois testes sorológicos, o ELISA e a imunofluorescência indireta. Uma amostra foi reagente no teste ELISA mas não reagente na imunofluorescência indireta, e 13 foram reativos na imunofluorescência indireta. Essas 14 amostras (31\%) foram submetidas ao teste TESA-blot, e todos deram negativos. Assim, nenhum dos casos suspeitos de CCC foi confirmado por método sorológico.

\section{Detecção e caracterização molecular do T. cruzi}

As amostras que apresentaram pelo menos uma reação sorológica positiva foram submetidas à análise molecular; dois foram positivas e identificadas como TcIII/IV (Figura 1).

Dados clínicos e laboratoriais desses dois pacientes estão resumidos na Tabela 2.

\section{Discussão}

\section{Prevalência da doença de Chagas}

Por muito tempo, acreditou-se que não existiam casos de DC na Amazônia brasileira. No entanto, isso mudou ao longo dos anos com relatos de muitos casos de DC aguda e crônica.

De acordo com o consenso brasileiro, o diagnóstico de DC deve ser confirmado pela combinação de dois métodos sorológicos. Estudos prévios realizados no estado do Amazonas ${ }^{14}$ relataram uma baixa prevalência de baixa morbidade da DC, e discutiram a provável baixa eficácia dos testes sorológicos comerciais disponíveis para as cepas circulantes, considerando que esses testes são realizados com diferentes cepas. ${ }^{11}$

Até o momento, foram relatados oito casos de $\mathrm{CCC}^{6-8}{ }^{6-8} \mathrm{e}$ quatro desses pacientes (50\%) apresentaram aneurisma apical, uma frequência esperada de acordo com estudos com pacientes de áreas endêmicas. ${ }^{15}$ Ferreira et al. ${ }^{8}$ estudaram pacientes com disfunção sistólica do ventrículo esquerdo de causa desconhecida, e encontraram uma prevalência de CCC de 8,1\%.

Em nosso estudo, os dois pacientes que apresentaram resultado de DNA positivo não apresentaram aneurisma, e um deles apresentou achados eletrocardiográficos normais. Um ECG normal não é comum (5\%) segundo estudos prévios, mas é possível mesmo na presença de uma doença cardíaca clínica ou disfunção no ecocardiograma. ${ }^{16,17}$ Vale destacar, no entanto, que a presença de DNA do T. cruzi pode não ser suficiente para confirmar CCC, e não podemos excluir uma possível coincidência na identificação do genótipo do parasita no paciente com disfunção ventricular grave, sem aneurisma apical ou diagnóstico sorológico, e com um ECG normal. O segundo paciente com DNA positivo apresentou bloqueio de ramo direito e bloqueio fascicular anterior esquerdo no ECG. Nesse paciente, a presença de DNA de T. cruzi e alterações típicas no ECG torna o diagnóstico de CCC possível mesmo sem diagnóstico sorológico. 


\section{Carta Científica}

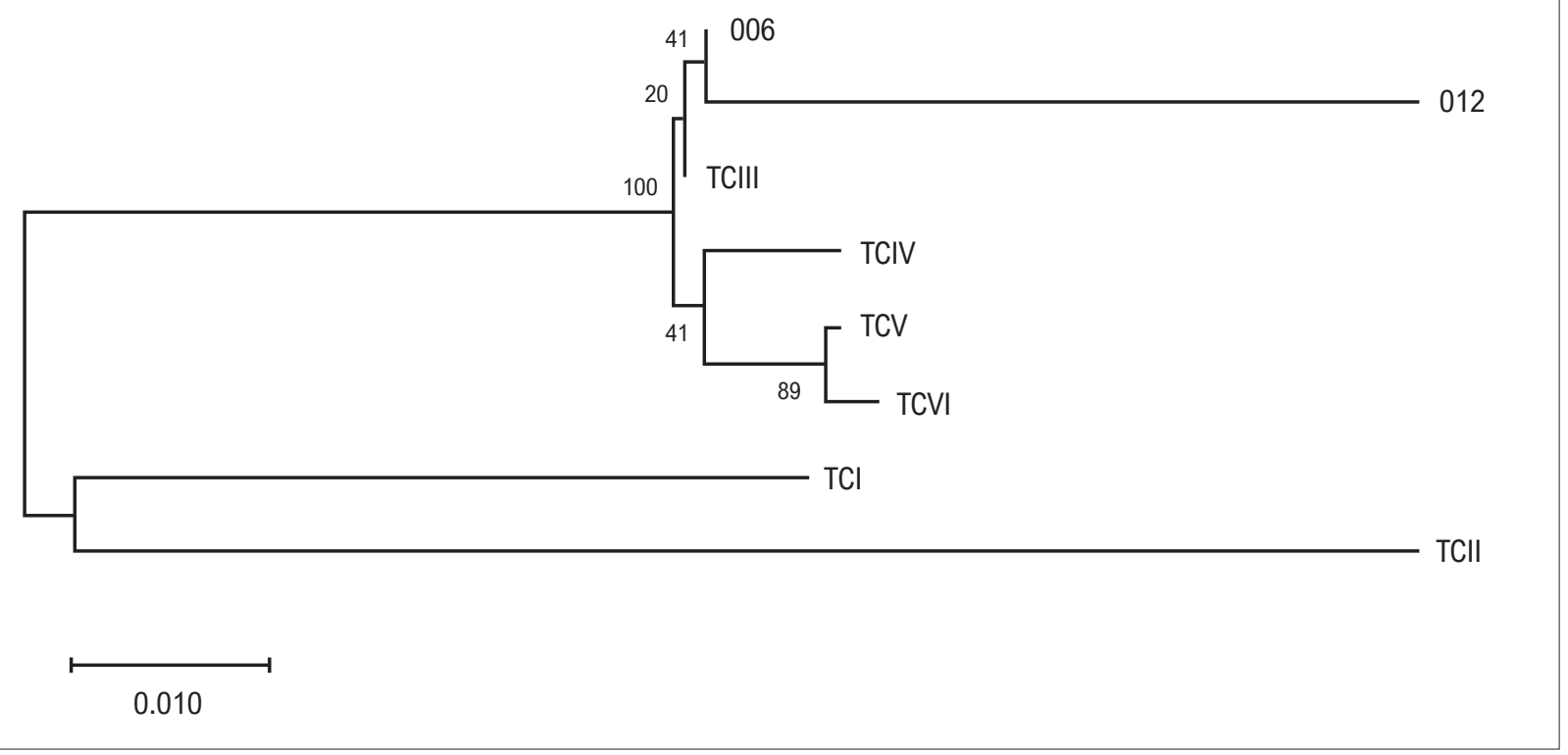

Figura 1 - Posição filogenética do Trypanosoma cruzi nas amostras 006 e 012 baseada nas sequências genéticas da subunidade Il da citocromo oxidase.

Embora o presente estudo não tenha identificado novos casos de CCC, como proposto inicialmente, nossos resultados levantam algumas questões: a doença de Chagas crônica, e consequentemente a CCC, é ainda subdiagnosticada na região da Amazônia brasileira? Os métodos sorológicos disponíveis têm se tornado menos eficiente em relação às cepas circulantes do parasita nesta região?

\section{Detecção e caracterização molecular do T. cruzi}

Apesar de técnicas moleculares não terem sido utilizadas no diagnóstico de DC devido a possíveis resultados falso positivos, o método foi incluído no estudo com base em estudos prévios que corroboram sua especificidade em infecções crônicas, e seu papel como um método complementar em casos em que resultados nos testes sorológicos persistirem inconclusivos. ${ }^{18}$ Nós identificamos o DNA do parasita em duas amostras, e determinamos seu genótipo como TcIII/TcIV.

Esta é a primeira vez em que a cepa TcllI/TcIV do T. cruzi é encontrada em uma fase crônica da doença. Este fato pode estar relacionado com a presença de Tclll nos vetores e reservatórios, e TcIV na fase aguda da DC, conforme relatado previamente na região. ${ }^{11,19}$ Outros estudos mostraram uma reatividade satisfatória ao teste sorológico e TESA-blot em regiões endêmicas no Brasil e no Panamá. ${ }^{9}$ No entanto, devido à região amazônica ainda ter uma epidemiologia particular, todos os recursos disponíveis foram usados neste estudo a fim de chegar a um resultado satisfatório para o paciente, incluindo uma PCR qualitativa reativa. ${ }^{20}$

\section{Limitações do estudo}

O pequeno tamanho amostral limitou nossa capacidade de demonstrar o real cenário da CCC em nossa região. Similarmente, temos visto uma baixa reatividade dos kits sorológicos comerciais disponíveis e uma reatividade cruzada moderada com outras doenças endêmicas.

\section{Conclusões}

Uma baixa prevalência de CCC foi observada neste grupo de participantes. Em duas das 45 amostras (4\%), fragmentos de DNA de T. cruzi foram detectados seguindo-se protocolos rígidos. No entanto, é importante lembrar que a maioria das amostras de soro de pacientes desta região tem baixa reatividade sorológica e moderada reatividade cruzada com outras doenças, o que torna difícil o diagnóstico. Assim, é muito importante a continuidade de pesquisas clínicas e epidemiológicas para determinar a real situação da CCC na região da Amazônia.

\section{Agradecimentos}

Gostaríamos de agradecer ao Laboratório de Protozoologia - Instituto de Medicina Tropical/SP pelo auxílio com o TESAblot, e ao Dr. George Villarouco pelo auxílio com as técnicas moleculares.

\section{Contribuição dos autores}

Concepção e desenho da pesquisa: Ortiz JV, BarbosaFerreira JMB; Obtenção de dados: Ortiz JV, Couceiro KN, Sousa DRT, Barbosa-Ferreira JMB; Análise e interpretação dos dados: Ortiz JV, Couceiro KN, Doria SS, Sousa DRT, Silveira HMC, Kesper Junior K, Barbosa-Ferreira JMB; Obtenção de financiamento: Barbosa-Ferreira JMB; Redação do manuscrito: Ortiz JV, Kesper Junior K; Revisão crítica do manuscrito quanto ao conteúdo intelectual importante: Couceiro KN, Silveira HMC, Guerra MGVB, Guerra JAO, Barbosa-Ferreira JMB.

\section{Potencial conflito de interesse}

Não há conflito com o presente artigo 
Tabela 1 - Características clínicas e demográficas de todos os casos suspeitos de cardiomiopatia chagásica

\begin{tabular}{lc} 
Variável & Total (n=45) \\
\hline Idade (anos) & $59,3 \pm 12,3$ \\
\hline Sexo & $35(77,8 \%)$ \\
\hline Masculino & $10(22,2 \%)$ \\
\hline Feminino & $34(75,6 \%)$ \\
\hline Área & $11(24,4 \%)$ \\
\hline Rural & $4(9,0 \%)$ \\
\hline Urbana & $32(71,1 \%)$ \\
\hline Origem & $1(2,2 \%)$ \\
\hline Acre & $6(13,3 \%)$ \\
\hline Amazonas & $2(4,4 \%)$ \\
\hline Maranhão &
\end{tabular}

Fatores de susceptibilidade

Agricultura/extrativismo/pescaria

Consumo de carne de caça

$32(71,1 \%)$

Consumo de fruto do açaizeiro

Hábito de entrar na floresta

Eletrocardiograma

Bloqueio de ramo direito

$2(4,4 \%)$

Bloqueio de ramo esquerdo

Bloqueio fascicular anterior esquerdo

Fibrilação atrial

Repolarização ventricular

Normal

\section{Ecocardiograma transtorácico}

FEVE $(\%)$

$30,3 \pm 10,7$

Diâmetro diastólico do ventrículo esquerdo (mm)

Acinesia localizada

Parede anterior septal

Parede lateral inferior

Parede septal inferior

Parede inferior

Hipocinesia difusa

Comorbidades

Diabetes mellitus

Hipertensão

Dados expressos em média \pm desvio padrão e porcentagens; FEVE: fração de ejeção do ventrículo esquerdo. 


\section{Carta Científica}

\section{Fontes de financiamento}

O presente estudo foi financiado pela Fundação de Amparo à Pesquisa do Estado do Amazonas (FAPEAM) Projeto Universal.

\section{Referências}

1. Chagas C. Nova Tripanozomiaze Humana: Estudos sobre a Morfolojia e o Ciclo Evolutivo do Schizotrypanum Cruzi n. gen., n. sp., Ajente Etiolojico de Nova Entidade Morbida do Homem. Mem Inst Oswaldo Cruz. 1909;1(2):159-218. doi: 10.1590/S0074-02761909000200008

2. World Health Organization. Integrating Neglected Tropical Diseases into Global Health and Development: Fourth WHO Reporton Neglected Tropical Diseases. Geneva: World Health Organization; 2017.

3. Vieira JL, Távora FRF, Sobral MGV, Vasconcelos GG, Almeida GPL, Fernandes JR, et al. Chagas Cardiomyopathy in Latin America Review. Curr Cardiol Rep. 2019;21(2):8. doi: 10.1007/s11886-019-1095-y.

4. Bestetti RB, Muccillo G. Clinical Course of Chagas' Heart Disease: A Comparison with Dilated Cardiomyopathy. Int J Cardiol. 1997;60(2):187-93. doi: 10.1016/ s0167-5273(97)00083-1.

5. Ferraroni J, MeloJN, Camargo M. Moléstia de Chagas na Amazônia: Ocorrência de Seis Casos Suspeitos, Autóctones, Sorologicamente Positivos. Acta Amaz. 1977;7(3):438-40.

6. Albajar PV, Laredo SV, Terrazas MB, Coura JR. Dilated Cardiomyopathy in Patients With Chronic Chagasic Infection: Report of Two Fatal Autochthonous Cases From Rio Negro, State of Amazonas, Brazil. Rev Soc Bras Med Trop. 2003;36(3):401-7.

7. Xavier SS, Sousa AS, Viñas PA, Junqueira AC, Bóia MN, Coura JR. Chronic Chagasic Cardiopathy in the Rio Negro, Amazon State. Report of Three New Autochthonous Cases Confirmed by Serology, Clinical Examination, Chest X-rays, Electro and Echocardiography. Rev Soc Bras Med Trop. 2006;39(2):2116. doi: 10.1590/s0037-86822006000200015.

8. Ferreira JM, Guerra JA, Magalhães BM, Coelho LI, Maciel MG, Barbosa M. Chronic Chagasic Cardiopathy in Amazon Region: An Etiology to Remember. Arq Bras Cardiol. 2009;93(6):93-109. doi: 10.1590/s0066782x2009001200028.

9. Caballero EZ, Correa R, Nascimento MS, Villarreal A, Llanes A, Kesper NJr. High Sensitivity and Reproducibility of in-house ELISAs Using Different Genotypes of Trypanosoma Cruzi. Parasite Immunol. 2019;41(7):e12627. doi: 10.1111/ pim.12627.

10. Umezawa ES, Nascimento MS, Kesper NJr, CouraJR, Borges-Pereira J, Junqueira AC, etal. ImmunoblotAssay Using Excreted-Secreted Antigens of Trypanosoma

\section{Vinculação acadêmica}

Este artigo é parte de dissertação de mestrado de Jessica Vanina Ortiz pela Universidade do Estado do Amazonas Programa de Pós-Graduação em Medicina Tropical.

Cruzi in Serodiagnosis of Congenital, Acute, and Chronic Chagas' Disease. J Clin Microbiol. 1996;34(9):2143-7. doi: 10.1128/jcm.34.9.2143-2147.1996.

11. Barbosa M, Ferreira JM, Arcanjo AR, Santana RA, Magalhães LK, Magalhães LK, et al. Chagas Disease in the State of Amazonas: History, Epidemiological Evolution, Risks of Endemicity and Future Perspectives. Rev Soc Bras Med Trop. 2015;48(Suppl 1):27-33. doi: 10.1590/0037-8682-0258-2013.

12. Freitas JM, Augusto-Pinto L, PimentaJR, Bastos-Rodrigues L, Gonçalves VF, Teixeira SM, etal. Ancestral Genomes, Sex, and the Population Structure of Trypanosoma Cruzi. PLoS Pathog. 2006;2(3):24. doi: 10.1371/journal.ppat.0020024.

13. Kumar S, Stecher G, Li M, Knyaz C, Tamura K. MEGA X: Molecular Evolutionary Genetics Analysis Across Computing Platforms. Mol Biol Evol. 2018;35(6):1547-9. doi: 10.1093/molbev/msy096.

14. Coura JR, Junqueira AC, Ferreira JMB. Surveillance of Seroepidemiology and Morbidity of Chagas Disease in the Negro River, Brazilian Amazon. Mem Inst Oswaldo Cruz. 2018;113(1):17-23. doi: 10.1590/0074-02760170169.

15. Prata A. Clinical and Epidemiological Aspects of Chagas Disease. Lancet Infect Dis. 2001;1(2):92-100. doi: 10.1016/S1473-3099(01)00065-2.

16. Rosenbaum MB, Alvarez AJ. The Electrocardiogram in Chronic Chagasic Myocarditis. Am Heart J. 1955;50(4):492-527. doi: 10.1016/00028703(55)90296-9.

17. Fernandez AB, Nunes MC, Clark EH, Samuels A, Menacho S, Gomez J, et al. Electrocardiographic and Echocardiographic Abnormalities in Chagas Disease: Findings in Residents of Rural Bolivian Communities Hyperendemic for Chagas Disease. Glob Heart. 2015;10(3):159-66. doi: 10.1016/j.gheart.2015.07.004.

18. Hernández C, Cucunubá Z, Flórez C, Olivera M, Valencia C, Zambrano P, et al. Molecular Diagnosis of Chagas Disease in Colombia: Parasitic Loads and Discrete Typing Units in Patients from Acute and Chronic Phases. PLoS Negl Trop Dis. 2016;10(9):e0004997. doi: 10.1371/journal.pntd.0004997.

19. Santana RA, Magalhães LK, Magalhães LK, Prestes SR, Maciel MG, Silva GA, et al. Trypanosoma Cruzi Strain TCl is Associated with Chronic Chagas Disease in the Brazilian Amazon. Parasit Vectors. 2014;7:267. doi: 10.1186/1756-3305-7-267.

20. Schijman AG. Molecular Diagnosis of Trypanosoma Cruzi. Acta Trop. 2018;184:59-66. doi: 10.1016/j.actatropica.2018.02.019. 